\title{
Lesiones deportivas en deportistas universitarios chilenos Sports injuries in Chilean university athletes
}

Claudia Danes-Daetz, Florencia Rojas Toro, Valentina Tapia Mendoza

Universidad de los Andes (Chile)

\begin{abstract}
Resumen. La epidemiología de las lesiones deportivas es el estudio de las variables que intervienen en la aparición de lesiones en la población y de su distribución. Este tiene como propósito último aportar información para establecer medidas de prevención justificadas. En Chile, la práctica deportiva ha aumentado en los últimos años. A pesar de esto, no existen antecedentes de estudios que analicen las lesiones deportivas. Este artículo pretende identificar la distribución de las lesiones en diferentes disciplinas deportivas de jóvenes universitarios chilenos. Se realizó un seguimiento a 84 deportistas de ambos géneros pertenecientes a selecciones universitarias de fútbol, básquetbol y vóleibol durante un periodo de 6 meses, registrando todas las lesiones ocurridas. En nuestro ambiente, la mayoría de las lesiones ocurren más frecuentemente en hombres. Son más frecuentes en la práctica del básquetbol (40,7\%), seguido del fútbol (35,2\%) y vóleibol (24,1\%). Afectan principalmente a las extremidades inferiores, especialmente a la articulación del tobillo, seguida por la rodilla y muñeca-mano. El tipo de lesión más común en esta investigación fue el esguince cápsulo-ligamentoso (40,7\%), seguida por las lesiones musculares $(16,7 \%)$. La cantidad de lesiones por sobreuso y de origen traumático fueron similares, siendo estas últimas asociadas principalmente a un mecanismo de contacto directo $(76,9 \%)$. Para esta población resultó más común lesionarse durante los entrenamientos que en los partidos.
\end{abstract}

Palabras claves: Epidemiología; Deportes; Lesión; Deportistas, Vigilancia de Lesiones.

\begin{abstract}
The epidemiology of sports injuries is the study of the variables that participate in the occurrence of injuries in a population, and their distribution. Its ultimate purpose is to provide information to establish justified prevention measures. In Chile, sports practice has increased in recent years. Despite this, there is no antecedent of studies that analyze sports injuries in Chile. This article aims to identify the distribution of injuries of young Chilean university students in different sports disciplines. A total of 84 athletes of both genders, belonging to university football, basketball, and volleyball teams were followed up for a period of 6 months, recording all the injuries that occurred. In our area, most injuries occur more frequently in men. They are more frequent in the practice of basketball $(40,7 \%)$, followed by soccer $(35,2 \%)$ and volleyball $(24,1 \%)$. They mainly affect the lower extremities, especially the ankle joint, followed by knee and wrist-hand. Capsule-ligament injuries (40,7\%) and muscle injuries (16,7\%) are the most common. The amount of injuries due to overuse and traumatic origin were similar, the latter being related mainly to a direct contact mechanism (76.9\%). For this population it was more common to be injured during training than in matches.
\end{abstract}

Key words: Epidemiology; Sports; Injury; Athletes, Vigilancia de Injury Surveillance.

\section{Introducción}

Actualmente, la práctica de ejercicio físico y actividades deportivas ha adquirido un importante desarrollo en nuestra sociedad, siendo uno de los principales elementos de ocupación del tiempo libre y ocio en la población (Moreno Pascual, Rodríguez Pérez, \& Seco Calvo, 2008). Esto conlleva un aumento en la cantidad de lesiones musculoesqueléticas entre los deportistas (Osorio Ciro, Clavijo Rodríguez, Arango V., Patiño Giraldo, \& Gallego Ching, 2007).

Con respecto a la población chilena, según la Encuesta Nacional de Actividad Física y Deporte 2016, se ha observado que la práctica deportiva ha aumentado en un $2,4 \%$ y el sedentarismo disminuido en un $2,6 \%$, en comparación con la encuesta realizada el 2012. De acuerdo con esto, se puede confirmar que ha ido aumentado el número de deportistas en Chile, careciendo de estudios que identifiquen las lesiones más comunes en esta población.

La epidemiología de las lesiones deportivas es el estudio de la distribución y de las variables que intervienen en la aparición de lesiones en la población. Los estudios epidemiológicos deben ir encaminados a identificar el perfil del sujeto más susceptible de ser afectado, las característi-

Fecha recepción: 20-10-19. Fecha de aceptación: 01-03-20 Claudia Danes Daetz

cddanes@miuandes.cl cas de la lesión y del medio en que se produce el accidente, así como del agente provocador (Moreno Pascual et al., 2008).

En el ámbito mundial existen diversas revisiones acerca de la incidencia de lesiones en distintos deportes. En una revisión de lesiones en fútbol profesional masculino (LópezValenciano et al., 2019) se describe una incidencia de 8,1 lesiones/1000 horas de exposición, siendo estos 10 veces más propensos a lesionarse durante los partidos. Las lesiones de extremidad inferior tuvieron las tasas de incidencia más altas (6,8 lesiones/1000 horas de exposición). El tipo de lesión más común fue músculo-tendínea (4,6 lesiones/1000 horas de exposición). En el básquetbol (Andreoli et al., 2018), la mayoría de las lesiones también ocurren en las extremidades inferiores $(63,7 \%)$, siendo las más frecuentes las lesiones de tobillo $(21,9 \%)$ y rodilla $(17,8 \%)$. Las lesiones de extremidad superior representan entre un $12-14 \%$ del total de lesiones. El tipo de lesión varía según la categoría etaria de estudio. Finalmente, para el vóleibol (Kilic, Maas, Verhagen, Zwerver, \& Gouttebarge, 2017), la incidencia de lesión va desde 1,7 a 10,7 lesiones/1000 horas de juego, comprometiendo mayormente tobillos, rodillas y hombros.

La comparación de los resultados de estudios epidemiológicos es difícil por las diferencias en las características de la población y en la forma de reportar los datos (Osorio Ciro et al., 2007). La gran variación entre las tasas de incidencia se explica entonces por las diferencias existentes entre los deportes, los países, el nivel competitivo, las edades y la metodología empleada en los estudios. Es por esto 
que existe la necesidad constante en la comunidad científica de realizar más estudios epidemiológicos que consideren todas estas variables, para mejorar el conocimiento sobre lesiones deportivas y validar la efectividad de las intervenciones preventivas.

En Sudamérica existe escasa literatura referente a este tema. Un estudio del perfil de lesiones de un equipo juvenil de primera división de fútbol en Brasil (Gonçalvesgon, et al., 2020) concluye que la tasa general de incidencia de lesiones fue de 1,86/1000 horas de exposición. En total se documentaron 187 lesiones en 122 jugadores $(65,2 \%)$ y se perdieron 4792 días de actividad deportiva en total. La mayoría fueron lesiones musculares sin contacto en el muslo y esguinces de tobillo. La incidencia de lesiones fue mayor durante los partidos que en el entrenamiento. En cuanto al básquetbol, existe un estudio también brasileño donde se describe la prevalencia de lesiones del equipo nacional durante el 2002 (Moreira, Gentil, \& de Oliveira, 2003). Contabilizaron un total de 6,37 lesiones/atleta/temporada, lesiones preponderantemente no traumáticas $(64,7 \%)$. La lesión más común fue esguince de tobillo (12,8\%), seguida por traumatismo en la mano $(8,8 \%)$. La localización más común fue en los miembros inferiores (48,0\%). Aún menor información se puede encontrar en la literatura acerca del vóleibol sudamericano. Un estudio en Colombia (específicamente la región de Cauca), caracteriza las lesiones deportivas en un periodo de un año y medio (Villaquirán, Portilla Dorado, \& Vernaza, 2016). Incluyeron deportistas de 25 disciplinas diferentes, donde el vóleibol fue el cuarto deporte con mayor cantidad de lesiones $(8,7 \%$ de los deportistas presentaron lesión). Otro estudio de la estadística de lesiones en Selecciones Masculinas Juveniles Argentinas de Voleibol indica una predominancia de lesiones tendinosas por encima de cualquier otro tipo, siendo las más frecuentes las del aparato extensor de la rodilla (42,6\% del total de lesiones) y en segundo lugar las tendinopatías del hombro (17,1\% del total) (Locaso, 2012).

Según nuestro conocimiento, no existe literatura donde existan registros de lesiones en el deporte y sus características en Chile. Entonces, el objetivo de este estudio es identificar la distribución de las lesiones en diferentes disciplinas deportivas de jóvenes universitarios chilenos. Esta información serviría para aportar a la literatura actual y para la futura creación y orientación de programas de prevención de lesiones, importante a la hora de lograr potenciar la actividad física en la población sin ser sometidos a mayor riesgo.

\section{Material y Método}

Población de estudio. Para este estudio se realizó el seguimiento de lesiones durante 6 meses a todos los deportistas de fútbol, vóleibol y básquetbol femenino y masculino pertenecientes a la selección de una universidad en la ciudad de Santiago de Chile.

Se incluyeron a todos los deportistas masculinos y femeninos de edad entre 18 y 25 años, que fueran seleccionados de esta universidad en las disciplinas escogidas. Se excluyeron a todos aquellos que presentaran cualquier lesión o cirugía que impida jugar o entrenar de manera regular durante el comienzo de la temporada deportiva 2017.

Por lo tanto, como se observa en la Figura 1, la muestra final constó de 84 deportistas, 50 hombres y 34 mujeres. En cuanto a las diferentes disciplinas deportivas practicadas, la muestra cuenta con 33 futbolistas, 19 voleibolistas y 32 basquetbolistas.

Tras una charla informativa, todos los deportistas que aceptaron ser participantes firmaron un consentimiento informado. Inmediatamente después, se guio a cada deportista para completar una encuesta, con el fin de recolectar la información relevante de: 1) antecedentes personales y datos antropométricos, 2) antecedentes deportivos y 3) lesiones actuales, previas y recurrentes.

Seguimiento de lesiones deportivas. Después de realizada la encuesta inicial, cada dos meses se contactó a cada uno de los deportistas, con el fin de identificar las lesiones deportivas ocurridas durante los 6 meses que duró la temporada. Se realizó así una encuesta electrónica adaptada ontenía preguntas basadas en una revisión acerca de la definición de lesiones y recolección de datos en futbolistas (Fuller et al., 2006). En el presente estudio se consideró como lesión a cualquier traumatismo musculoesquelético que requirió intervención médica y resultó en una pérdida de más de 1 día de la participación en actividades deportivas (Kerr, Collins, Fields, \& Comstock, 2011). Los deportistas que no respondieron el correo fueron contactados directamente vía telefónica, logrando así las respuestas del total de la muestra.

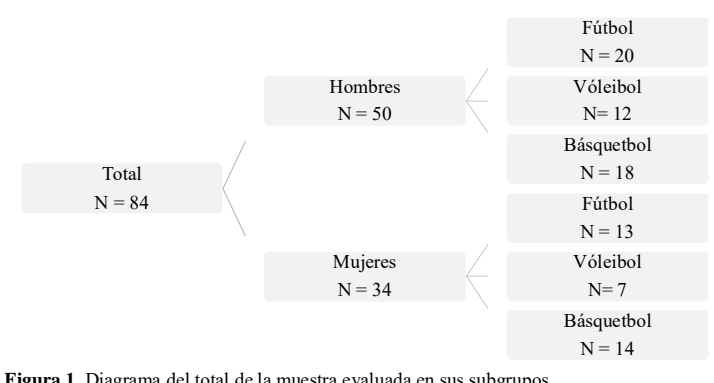

Resultados

\section{Caracterización de la muestra}

La muestra analizada correspondió a 84 deportistas, incluyendo las tres disciplinas deportivas en ambos géneros. Los datos demográficos son presentados en la Tabla 1.

\begin{tabular}{|c|c|c|c|}
\hline & $\begin{array}{c}\text { Hombres } \\
(\mathrm{n}=50)\end{array}$ & $\begin{array}{c}\text { Mujeres } \\
(\mathrm{n}=34)\end{array}$ & $\begin{array}{c}\text { Total } \\
(\mathrm{n}=84)\end{array}$ \\
\hline$\overline{E d a d ~(a n ̃ o s) ~}$ & $20,32 \pm 1,70$ & $20,47 \pm 1,74$ & $20,38 \pm 1,71$ \\
\hline Masa (kg) & $75,28 \pm 10,44$ & $62,65 \pm 8,38$ & $70,17 \pm 11,45$ \\
\hline Talla (m) & $1,81 \pm 0,08$ & $1,65 \pm 0,07$ & $1,74 \pm 0,11$ \\
\hline IMC $\left(\mathrm{kg} / \mathrm{m}^{2}\right)$ & $22,99 \pm 2,29$ & $22,94 \pm 2,61$ & $22,97 \pm 2,41$ \\
\hline
\end{tabular}

\section{Descripción de lesiones ocurridas:}

De los 84 deportistas evaluados, 41 de ellos $(48,8 \%)$ refirieron haber sufrido alguna lesión en el periodo competitivo entre abril-septiembre 2017. En total ocurrieron 54 lesiones, ya que algunos presentaron 2 o incluso 3 lesiones diferentes durante este periodo.

En cuanto a la distribución de las lesiones por sexo (Figura 2), en este estudio se observa que, en términos absolutos, las lesiones son más frecuentes en hombres con 36 ca$\operatorname{sos}(67,0 \%)$ que en mujeres con $18 \operatorname{casos}(33,0 \%)$. En cuanto 
al porcentaje de deportistas lesionados en función del sexo, un $52,0 \%$ de los hombres evaluados se lesionaron durante el estudio (26 lesionados del total de 50 hombres), de los cuales 8 presentaron doble lesión y uno incluso llegó a lesionarse tres veces en el mismo periodo. Por otro lado, un 44,1\% de las mujeres incluidas en la muestra presentaron lesiones (15 lesionadas de 34 mujeres en total), de las cuales solo 3 tuvieron doble lesión.

Por otro lado, la disciplina con mayor número de lesiones presentadas por los deportistas fue el básquetbol, causante de 22 lesiones (40,7\%), seguido por el fútbol con 19 lesiones $(35,2 \%)$, y finalmente el vóleibol con 13 lesiones (24,1\%), mostrado en la Figura 3. En función del número de participantes lesionados, el básquetbol continúa en primer lugar con un $56,3 \%$ de sus deportistas lesionados al menos en una ocasión, luego con un $52,6 \%$ el vóleibol y finalmente con un $39,4 \%$ el fútbol.

En cuanto a la localización anatómica de las lesiones encontradas en este estudio, casi dos tercios de las lesiones $(63,0 \%)$ ocurrieron en las extremidades inferiores. Las extremidades superiores concentran casi por completo el tercio restante $(31,5 \%)$, mientras que solo una pequeña parte $(5,5 \%)$ afectaron al tronco. En la Figura 4 se muestra en detalle la frecuencia de localización anatómica de las lesiones en este estudio. La articulación del tobillo ha sido marcadamente más frecuente $(24,1 \%)$ que cualquier otra. Le siguen las articulaciones de rodilla y muñeca/mano, ambas con un $14,8 \%$ de frecuencia. Luego vendrían las articulaciones que unen al aparato axial, hombro $(13,0 \%)$ y cadera $(9,3 \%)$, acompañado de las regiones del muslo $(9,3 \%)$ y pierna $(3,7 \%)$. Recién aquí

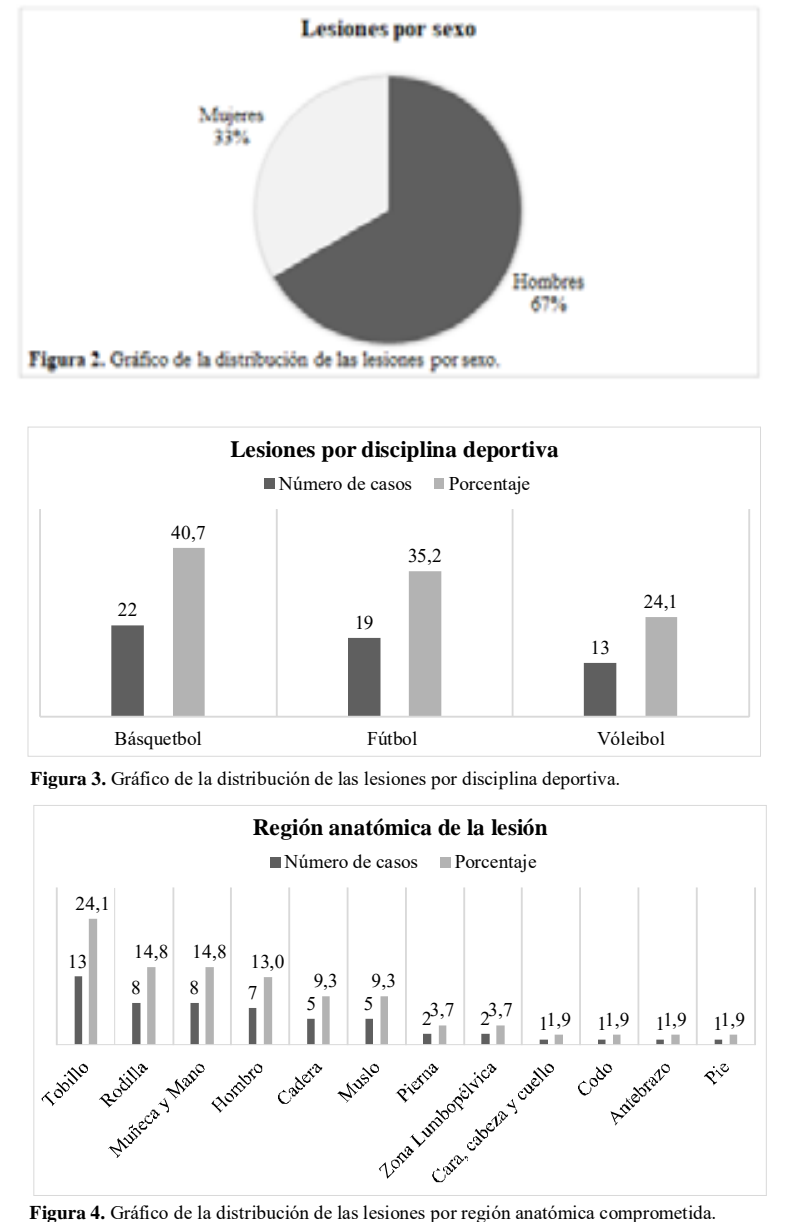

encontramos lesiones en la determinada zona lumbopélvica $(3,7 \%)$, dejando finalmente con solo un episodio de lesión a las regiones anatómicas de cara/cabeza/cuello, codo, antebrazo y pie ( $1,9 \%$ cada uno).

El tipo de lesión más común en esta investigación fue el esguince de ligamentos o cápsula, el cual representa al 40,7\% de las lesiones registradas. La segunda lesión más predominante vendría siendo las que comprometen al tejido muscular, con un $16,7 \%$. Entre las lesiones ligamentosas y musculares, suman más de la mitad de todas las lesiones ocurridas durante esta investigación. Luego vienen las lesiones óseas $(11,1 \%)$ y tendinosas $(9,3 \%)$. Las lesiones de menisco o cartílago $(3,7 \%)$, concusiones $(1,9 \%)$ y lesión de nervios $(1,9 \%)$ son las menos frecuente. Finalmente, 8 lesiones corresponden a otros tejidos afectados $(14,8 \%)$. Si se analiza de manera aislada por disciplina deportiva, en el fútbol existe una preponderancia de lesiones de ligamentos o esguinces (42,1\%), seguido por lesiones musculares $(21,1 \%)$. En el caso del vóleibol, también se presentó un predominio de los esguinces $(38,5 \%)$, pero en segundo lugar se encuentran tanto las lesiones musculares como las tendinopatías, con un 23,1\%. Finalmente, en el básquetbol, tal como en los otros deportes, los esguinces representan un $27,3 \%$ de las lesiones, siendo el tipo de lesión más común en este deporte. En el básquetbol, 5 lesiones $(22,7 \%)$ corresponden a «otras» lesiones, y un $13,6 \%$ correspondieron a fracturas (de mano y nasal).

En cuanto a la etiología, las lesiones se podrían separar por tener un origen traumático o por sobrecarga (Chéron, Le Scanff, \& Leboeuf-Yde, 2017). Para este estudio, las lesiones por sobrecarga alcanzaron un $51,9 \%$ del total de las lesiones, superando solo por un pequeño porcentaje a las lesiones causadas por un origen traumático, las que representan un $48,1 \%$.

En cuanto al contexto de la lesión, las lesiones de origen traumático o agudas se pueden clasificar como mecanismo por contacto directo o por no contacto (indirecto) (Butler, Lehr, Fink, Kiesel, \& Plisky, 2013; Kerr et al., 2011). En este estudio cercano a tres cuartos de las lesiones $(76,9 \%)$ fueron causadas por contacto o colisión, ya sea con otro jugador, el balón u otro objeto. Las lesiones por no contacto o mecanismo indirecto correspondió entonces a un $23,1 \%$ de las lesiones traumáticas.

En otro ámbito, la mayoría de las lesiones ocurrieron durante los entrenamientos $(61,1 \%)$, y un porcentaje menor durante los partidos (31,5\%). El 7,4\% restante corresponden a lesiones donde el deportista no logró identificar específicamente el momento de inicio de la lesión, pero que comprometió funcionalmente al voluntario para poder realizar su deporte. Si se analiza separado por disciplina deportiva, existe una gran diferencia entre los tres deportes. En el caso del fútbol, el 50\% de las lesiones ocurrieron durante los entrenamientos, y la otra mitad durante partidos. En el caso del vóleibol, todas de las lesiones ocurrieron durante la práctica, no habiendo registro de lesiones durante alguna competencia. Finalmente, el básquetbol presentó un $60 \%$ de lesiones durante el entrenamiento y el $40 \%$ restantes sucedieron durante el desarrollo de un partido.

Dentro del registro de los diagnósticos médicos específicos de las lesiones, encontramos que los esguinces de tobillo grado I y grado II son los diagnósticos más recurrentes, 
tanto de tobillo izquierdo como derecho. También existió un caso de pinzamiento de tobillo derecho. Se presentaron diagnósticos relacionadas a sobrecarga, como la periostitis tibial $\mathrm{y}$ fascitis plantar. A nivel de rodilla, existieron muchas lesiones de etiología diferente: desde luxaciones y tendinopatías patelares a meniscopatía y síndrome de fricción de la banda iliotibial. Los desgarros registrados fueron catalogados con diferentes nomenclaturas, como desgarro, distensión o microdesgarro muscular, refiriéndose a la lesión de, en este caso, musculatura isquiotibial. A nivel de las articulaciones que unen las extremidades al aparato axial, encontramos un desgarro de labrum de cadera izquierda y un SLAP de hombro derecho. También refirieron un diagnóstico de discopatía con HNP lumbar. En los miembros superiores, un deportista refirió presentar una subluxación de nervio cubital, mientras que la mayoría presentaron lesiones de tipo esguinces de dedos. También existió una fractura de la falange media en la mano izquierda de un deportista. Otra fractura registrada fue un caso de fractura nasal.

\section{Discusión}

\section{Distribución por sexo}

Existe diversas referencias respecto a la relación entre el sexo y la aparición de lesiones deportivas (Brant, Johnson, Brou, Comstock, \& Vu, 2019; Flaxman, Smith, \& Benoit, 2014; Tenforde, Sayres, McCurdy, Sainani, \& Fredericson, 2013). En la distribución de las lesiones por sexo de este estudio se observa que, en términos absolutos, las lesiones son más frecuentes en hombres (67\%) que en mujeres (33\%). Esto se contradice con lo expuesto en la literatura actual, donde se expone más comúnmente un mayor predominio de lesiones en mujeres que en hombres (Brant et al., 2019; Carter, Westerman, \& Hunting, 2011; Sánchez Jover, F. y Gómez Conesa, 2008; Tenforde et al., 2013). Parte de esta diferencia en el riesgo de lesión podría explicarse por las diferencias anatómicas (Schilaty, Bates, Nagelli, Krych, \& Hewett, 2018; Wahl, Westermann, Blaisdell, \& Cizik, 2012), hormonales (Tenforde et al., 2013) y neuromusculares entre ambos géneros (Bahr \& Krosshaug, 2005; Flaxman et al., 2014), donde las mujeres presentan mayores factores de riesgo para las lesiones. Aun así existen algunos estudios donde los hombres presentan mayor cantidad de lesiones que las mujeres (Edouard, Branco, \& Alonso, 2016; García González, Albaladejo Vicente, Villanueva Orbáiz, \& Navarro Cabello, 2015). Esto sucede debido a que en general existen mayor cantidad de deportistas varones que damas y por lo tanto mayor exposición, tal como sucedió con esta muestra. Existen estudios que incluso no encontraron relación alguna entre la ocurrencia de lesión y género del deportista (Prieto Andreu, 2015).

\section{Cantidad de lesiones por disciplina deportiva}

Se ha visto que un mayor número de lesiones totales se asociaron con deportes colectivos, como fútbol y básquetbol, en comparación a deportes individuales (Prieto Andreu, 2015). La disciplina deportiva con mayor número de deportistas lesionados en esta muestra fue el básquetbol, seguido por el fútbol y finalmente el vóleibol. Existe un estudio que analizó los mismos tres deportes en deportistas femeninas, cuyos resultados exponen al fútbol en primer lugar como deporte más lesivo, seguido por el vóleibol y finalmente el básquetbol (Foss, Myer, \& Hewett, 2014). Aun así, el fútbol y el básquetbol son los deportes más frecuentemente implicados como causantes de lesiones en Europa (Al Bimani et al., 2018; García González et al., 2015; Osorio Ciro et al., 2007; Ríos Azuara, D.; Pérez Flores, D. y Ríos Alcolea, 2014). Un estudio ya había obtenido resultados símiles al presente estudio, identificando mayor cantidad de lesiones ocurridas en el básquetbol que en el fútbol (Carter et al., 2011). La alta incidencia de lesiones en deportes como el básquetbol y fútbol es en parte atribuible a la gran popularidad alcanzada por estos deportes, donde la población practicante llega a ser superior a otras disciplinas (Carter et al., 2011; Moreno Pascual et al., 2008). Cuando se ajustan los resultados en relación con el número de practicantes y el tiempo de exposición, disciplinas como el fútbol americano o rugby presenta un riesgo lesional hasta dos veces mayor que el fútbol (Carter et al., 2011; Kaplan, Goodwillie, Strauss, \& Rosen, 2008). El vóleibol, por otra parte, es un deporte de no contacto, por lo tanto tiende a presentarse como una disciplina relativamente más segura (Reeser, Verhagen, Briner, Askeland, \& Bahr, 2006).

\section{Localización anatómica de las lesiones}

Distintos estudios confirman que la mayoría de las lesiones de deportes colectivos afectan a los miembros inferiores (García González et al., 2015; Hootman, Dick, \& Agel, 2007; Penichet-Tomás, Becerra, Jiménez-Olmedo, Pueo, \& Agulló, 2019). Esto concuerda con los resultados encontrados en este estudio, donde casi dos tercios de las lesiones ocurrieron en las extremidades inferiores.

Específicamente la articulación de tobillo es la más afectada en cantidad de lesiones según la literatura actual, seguida por la rodilla (Bere, Kruczynski, Veintimilla, Hamu, \& Bahr, 2015; Foss et al., 2014; Hootman et al., 2007; Sánchez Jover, F. y Gómez Conesa, 2008; Toro et al., 2020). Esto concuerda con los hallazgos de esta investigación. El hecho de que la muñeca/mano haya presentado una alta frecuencia, similar a las lesiones de rodilla, puede ser debido a la naturaleza de los deportes estudiados en esta ocasión. Tanto el básquetbol como el vóleibol presentan una alta incidencia de lesiones de muñeca/mano (Bere et al., 2015; Reeser et al., 2006; Ríos Azuara, D.; Pérez Flores, D. y Ríos Alcolea, 2014).

\section{Tipos de lesiones}

Entre las lesiones ligamentosas y musculares estudiadas en esta población, suman más de la mitad de todas las lesiones ocurridas durante la investigación. En la mayoría de los estudios publicados, las lesiones ligamentosas son las más frecuentes (Bere et al., 2015; Carter et al., 2011; Doherty et al., 2014; Kerr et al., 2011; Toro et al., 2020). El esguince, tiende a representar entre el $25 \%$ y el $35 \%$ de las lesiones (Foss et al., 2014; Kerr et al., 2011; Sánchez Jover, F. y Gómez Conesa, 2008). En esta población de estudio, los esguinces superan incluso estos índices, llegando a 40,7\% de las lesiones registradas. Además, son el tipo de lesión más preponderante para cada una de las disciplinas deportivas estudiadas de manera separada.

Las lesiones musculares también tienden a presentar una 
alta frecuencia, llegando a ser incluso más frecuentes que los esguinces en algunos estudios (Bere et al., 2015; Edouard et al., 2016; Renshaw \& Goodwin, 2016; Zafra, 2009). En estos estudios, las lesiones musculares representan entre el 10 y el 25\% (Bere et al., 2015; Foss et al., 2014; Kerr et al., 2011), aunque en deportes como el fútbol pueden llegar al $40 \%$ (Edouard et al., 2016; Renshaw \& Goodwin, 2016; Zafra, 2009). En este estudio, 4 de los 9 desgarros fueron reportados por futbolistas, siendo el deporte con mayor registro de este tipo de lesión. Aun así, los desgarros alcanzaron solo un $21 \%$ de ocurrencia entre las lesiones reportadas en esta disciplina, siendo un valor menor al registrado en la literatura para esta especialidad deportiva.

\section{Etiología de la lesión}

En este estudio tanto las lesiones traumáticas como por sobreuso tuvieron una presentación similar, aunque las lesiones por sobrecarga fueron ligeramente más frecuentes. Esto se contrapone a los resultados preponderantes en la literatura, donde las lesiones agudas suelen ser más comunes (Yang et al., 2012).

Según estudios relacionados al fútbol, la incidencia de lesiones traumáticas (5,9 lesiones/1000 horas de exposición) alcanza a ser el doble que la de lesiones por sobreuso $(2,4$ lesiones/ 1000 horas de exposición) (López-Valenciano et al., 2019). En el básquetbol, también suele existir un predominio de las lesiones agudas, siendo su incidencia de 6,0/1000 horas y los esguinces de tobillo la lesión traumática más común (Cumps, Verhagen, \& Meeusen, 2007). La incidencia de lesiones por sobreuso registradas para este deporte es de 3,8/ 1000 horas, siendo la rodilla la zona mayormente afectada. Las investigaciones epidemiológicas han revelado que las voleibolistas, en similar situación al básquetbol, tienden a sufrir más comúnmente esguinces agudos de tobillo, seguido por condiciones de sobreuso de la rodilla, hombro y tronco (Reeser et al., 2006). Debido al gran volumen de saltos y movimientos repetitivos a los que están sometidos en estos deportes, tienden a sobrecargar estas articulaciones (Bere et al., 2015; Reeser et al., 2006).

El hecho de que en estos deportes se registre una mayor incidencia de lesiones traumáticas que de sobrecarga puede tener relación a que tradicionalmente, la mayoría de los estudios de lesiones utilizan la pérdida de tiempo del deporte como criterio principal para definir la ocurrencia de la lesión. Pero este enfoque subestima el impacto de las lesiones por sobrecarga ya que los atletas con una lesión por uso excesivo a menudo pueden seguir entrenando y competir a pesar de los síntomas y limitaciones persistentes asociados con las lesiones (Clarsen et al., 2020). Las lesiones por sobreuso suelen ser más preponderantes en mujeres que hombres, y están directamente relacionadas a la cantidad de actividad física que se practique (Cuff, Loud, \& O'Riordan, 2010).

\section{Contexto de la lesión}

Las lesiones traumáticas pueden estar encasilladas en el contexto de la lesión, pudiendo ser por contacto y por no contacto (Butler et al., 2013; Kerr et al., 2011). En los resultados de este estudio, las lesiones por contacto o con mecanismo directo fueron tres veces más frecuentes que las indirectas. Las lesiones por contacto suelen ser más preponde- rantes en deportes cuyas reglas permiten constante contacto físico directo entre jugadores, como el fútbol y baloncesto (Kerr et al., 2011). Las lesiones agudas de no contacto incluyen lesiones como esguinces ligamentosos o desgarros musculares, donde no existe contacto alguno con un tercero en su mecanismo lesional.

A pesar de que el vóleibol es considerado como deporte de no contacto, la lesión más común es el esguince lateral de tobillo, el que suele estar más comúnmente relacionado con un mecanismo directo. En el vóleibol indoor, los esguinces de tobillo ocurren con mayor frecuencia en la red, como resultado del contacto entre el atacante y los bloqueadores del equipo contrario en la línea central (Reeser et al., 2006). Este mismo mecanismo de lesión se repite para los esguinces de tobillo reportados por basquetbolistas, predominando el contacto al caer sobre el pie del oponente (Cumps et al., 2007). En el caso del fútbol, ser tacleado o chocar con un oponente parecen ser los incidentes lesionales más comunes, representando casi un $50 \%$ de todas las lesiones traumáticas, seguido de lesiones causadas por acciones de no contacto, como acelerar o cambiar de dirección (LópezValenciano et al., 2019). Estos resultados concuerdan con lo reportado en este estudio.

En la literatura, se identifica que las lesiones suceden con mayor frecuencia durante los partidos (Hootman et al., 2007; Penichet-Tomás et al., 2019; Sánchez Jover, F. y Gómez Conesa, 2008). Algunos autores lo asocian al tipo de deporte, donde deportes de contacto como el fútbol suelen aumentar su frecuencia de lesión debido al contacto más agresivo contra los contrincantes en los partidos, contacto que se suele restringir durante los entrenamientos. Esto sumado a que la exigencia física exigida durante un partido es percibida como mayor al compararlo con un entrenamiento (Campos \& Toscano, 2018). En la contraparte, deportes que no se asocian tradicionalmente a contacto significativo con el contrincante, como el vóleibol, suelen no presentar diferencias tan dramáticas entre las tasas de lesión en entrenamiento y partidos (Hootman et al., 2007). Esto podría explicar los resultados de este y otros estudios, donde se encontraron mayor predominancia de las lesiones durante los entrenamientos (Renshaw \& Goodwin, 2016; Zafra, 2009).

\section{Limitaciones}

La investigación en epidemiología de lesiones deportivas presenta importantes inconvenientes en general, partiendo por las distintas definiciones existentes de una «lesión deportiva», y de las características de la recolección de datos. La mayor limitación de este estudio en particular es que la muestra estaba limitada a los deportistas de las selecciones de solo una universidad. A pesar de que, de nuestro conocimiento, este es el primer estudio de lesiones deportivas que incluye a voluntarios chilenos, estos resultados no se podrían extrapolar a toda la población. Otra limitación respecta al periodo de seguimiento, el cual fue de 6 meses. Se eligió este periodo ya que corresponde a la temporada deportiva a la que están sujetos los deportistas, y además de esta manera se minimizó a cero las pérdidas de voluntarios propias de un estudio prospectivo. 


\section{Conclusión}

En este estudio, la mayoría de las lesiones ocurren en la práctica del básquetbol, seguido del fútbol y vóleibol, y afectan a las extremidades inferiores, especialmente a la articulación del tobillo. Las lesiones de ligamentos y las lesiones musculares son las más frecuentes, y se presentan más comúnmente en hombres que en mujeres, en términos absolutos.

Con relación a deportistas de nivel competitivo, el conocimiento de las lesiones más recurrentes podría dar lugar a programas de prevención específicos que disminuyan la tasa de lesiones y mejoren el rendimiento general del atleta.

\section{Conflicto de intereses}

Los autores declaran no tener conflicto de interés.

\section{Referencias}

Al Bimani, S. A., Gates, L. S., Warner, M., Ewings, S., Crouch, R., \& Bowen, C. (2018). Characteristics of patients with ankle sprain presenting to an emergency department in the south of England (UK): A seven-month review. International Emergency Nursing, 41, 38-44. https:// doi.org/10.1016/j.ienj.2018.05.008

Andreoli, C. V., Chiaramonti, B. C., Buriel, E., Pochini,A. D. C., Ejnisman, B., \& Cohen, M. (2018). Epidemiology of sports injuries in basketball: Integrative systematic review. BMJ Open Sport and Exercise Medicine, 4(1), 1-9. https:// doi.org/10.1136/bmjsem-2018-000468

Bahr, R., \& Krosshaug, T. (2005). Understanding injury mechanisms: a key component of preventing injuries in sport. British Journal of Sports Medicine, 39(6), 324 329. https://doi.org/10.1136/bjsm.2005.018341

Bere, T., Kruczynski, J., Veintimilla, N., Hamu, Y., \& Bahr, R. (2015). Injury risk is low among world-class volleyball players: 4-year data from the FIVB Injury Surveillance System. British Journal of Sports Medicine, 49(17), 1132-1137.https://doi.org/10.1136/bjsports-2015-094959

Brant, J. A., Johnson, B., Brou, L., Comstock, R. D., \& Vu, T. (2019). Rates and Patterns of Lower Extremity Sports Injuries in All Gender-Comparable US High School Sports. Orthopaedic Journal of Sports Medicine, 7(10), $232596711987305 . \quad$ https://doi.org/10.1177/ 2325967119873059

Butler, R. J., Lehr, M. E., Fink, M. L., Kiesel, K. B., \& Plisky, P. J. (2013). Dynamic Balance Performance and Noncontact Lower Extremity Injury in College Football Players: An Initial Study. Sports Health, 5(5), 417-422. https://doi.org/ $10.1177 / 1941738113498703$

Campos, M. Á. C., \& Toscano, F. J. T. (2018). Comparación de la percepción subjetiva del esfuerzo entre partidos amistosos y diferentes tipos de sesión en futbolistas profesionales. Retos: Nuevas Tendencias En Educación Física, Deporte y Recreación, 2041(34), 66-70.

Carter, E.A., Westerman, B. J., \& Hunting, K. L. (2011). Risk of injury in basketball, football, and soccer players, ages 15 years and older, 2003-2007. Journal of Athletic Training, 46(5), 484-488. https://doi.org/10.4085/1062-
6050-46.5.484

Chéron, C., Le Scanff, C., \& Leboeuf-Yde, C. (2017). Association between sports type and overuse injuries of extremities in adults: A systematic review. Chiropractic and Manual Therapies, 25(1), 4. https://doi.org/10.1186/ s12998-017-0135-1

Clarsen, B., Bahr, R., Myklebust, G., Andersson, S. H., Docking, S. I., Drew, M., ... Nabhan, D. (2020). Improved reporting of overuse injuries and health problems in sport/ : an update of the Oslo Sport Trauma Research Center questionnaires, 1-7. https://doi.org/10.1136/bjsports2019-101337

Cuff, S., Loud, K., \& O’Riordan, M.A. (2010). Overuse injuries in high school athletes. Clinical Pediatrics, 49(8), 731-736. https://doi.org/10.1177/0009922810363154

Cumps, E., Verhagen, E., \& Meeusen, R. (2007). Prospective epidemiological study of basketball injuries during one competitive season: Ankle sprains and overuse knee injuries. Journal of Sports Science and Medicine, 6(2), 204-211.

Doherty, C., Delahunt, E., Caulfield, B., Hertel, J., Ryan, J., \& Bleakley, C. (2014). The Incidence and Prevalence of Ankle Sprain Injury: A Systematic Review and Meta-Analysis of Prospective Epidemiological Studies. Sports Medicine, 44(1), 123-140. https://doi.org/10.1007/s40279-013$0102-5$

Edouard, P., Branco, P., \& Alonso, J.-M. (2016). Muscle injury is the principal injury type and hamstring muscle injury is the first injury diagnosis during top-level international athletics championships between 2007 and 2015. British Journal of Sports Medicine, 50(10), 619-630. https:// doi.org/10.1136/bjsports-2015-095559

Flaxman, T. E., Smith, A. J. J., \& Benoit, D. L. (2014). Sexrelated differences in neuromuscular control: Implications for injury mechanisms or healthy stabilisation strategies? Journal of Orthopaedic Research, 32(2), 310-317. https:/ /doi.org/10.1002/jor.22510

Foss, K. D. B., Myer, G. D., \& Hewett, T.E.(2014). Epidemiology of Basketball, Soccer, and Volleyball Injuries in MiddleSchool Female Athletes. The Physician and Sportsmedicine, 42(2), 146-153. https://doi.org/10.3810/ psm.2014.05.2066

Fuller, C. W., Ekstrand, J., Junge, A., Andersen, T. E., Bahr, R., Dvorak, J., ... Meeuwisse, W. H. (2006). Consensus statement on injury definitions and data collection procedures in studies of football (soccer) injuries. Scandinavian Journal of Medicine and Science in Sports, 16(2), 83-92. https://doi.org/10.1111/j.16000838.2006.00528.x

García González, C., Albaladejo Vicente, R., Villanueva Orbáiz, R., \& Navarro Cabello, E. (2015). Deporte de ocio en EspanPa. epidemiologiìa de las lesiones y sus consecuencias. Apunts. Educación Física y Deportes, 1(119), 62-70. https://doi.org/http://dx.doi.org/10.5672/ apunts.2014-0983.es.(2015/1).119.03

Gonçalvesgon , L., Cezarino, G., Bruno, ;, Da, L., Grüninger, S., Grüninger, G., \& Silva, R. S. (2020). Injury Profile in a Brazilian First-Division Youth Soccer Team: A Prospective Study. Journal of Athletic Training, 55(3). https:// doi.org/10.4085/1062-6050-449-18 
Hootman, J. M., Dick, R., \& Agel, J. (2007). Epidemiology of collegiate injuries for 15 sports: Summary and recommendations for injury prevention initiatives. Journal of Athletic Training. https://doi.org/10.1111/ j.1600-0838.2006.00528.x

Kaplan, K., Goodwillie, A., Strauss, E., \& Rosen, J. (2008). Rugby Injuries - A review of concepts and current literature. Bulletin of the NYU Hospital for Joint Diseases, 66(2), 86-93.https://doi.org/10.1136/bmj.304.6819.122-b

Kerr, Z. Y., Collins, C. L., Fields, S. K., \& Comstock, R. D. (2011). Epidemiology of player-player contact injuries among US high school athletes, 2005-2009. Clinical Pediatrics, 50(7), 594-603. https://doi.org/10.1177/ 0009922810390513

Kilic, O., Maas, M., Verhagen, E., Zwerver, J., \& Gouttebarge, V. (2017). Incidence, aetiology and prevention of musculoskeletal injuries in volleyball: Asystematic review of the literature. European Journal of Sport Science, 17(6), 765-793. https://doi.org/10.1080/ 17461391.2017.1306114

Locaso, F. J. (2012). Estadística de lesiones en Selecciones Masculinas Juveniles Argentinas de Voleibol. Rev. Asoc. Argent. Traumatol. Deporte, 19(1), 32-44.

López-Valenciano, A., Ruiz-Pérez, I., Garcia-Gómez,A., VeraGarcia, F. J., De Ste Croix, M., Myer, G. D., \& Ayala, F. (2019). Epidemiology of injuries in professional football: A systematic review and meta-analysis. British Journal of Sports Medicine, 1-9. https://doi.org/10.1136/bjsports2018-099577

Moreira, P., Gentil, D., \& de Oliveira, C. (2003). Prevalence of injuries of Brazilian Basketball National Team during 2002 season. Revista Brasileira De Medicina Do Esporte, 9(5), 263-266.

Moreno Pascual, C., Rodríguez Pérez, V., \& Seco Calvo, J. (2008). Epidemiología de las lesiones deportivas. Fisioterapia,30(1), 40-48. https://doi.org/10.1016/S02115638(08)72954-7

Osorio Ciro, J. A., Clavijo Rodríguez, M. P., Arango V., E., Patiño Giraldo, S., \& Gallego Ching, I. C. (2007). Lesiones deportivas. Iatreia. https://doi.org/10.1017/ CBO9781107415324.004

Penichet-Tomás, A., Becerra, M. O., Jiménez-Olmedo, J. M., Pueo, B., \& Agulló, J. J. E. (2019). Incidence of injury in elite Spanish beach handball players. Retos, 2041(36), 83-86.

Prieto Andreu, J. (2015). Variables deportivas y personales en la ocurrencia de lesiones deportivas. Diferencias entre deportes individuales y colectivos. Retos: Nuevas Tendencias En Educación Física, Deporte y Recreación, 2041(28), 21-25.

Reeser, J. C., Verhagen, E., Briner, W. W., Askeland, T. I., \& Bahr, R. (2006). Strategies for the prevention of volleyball related injuries. British Journal of Sports Medicine, 40(7), 594-600; discussion 599-600. https://doi.org/10.1136/ bjsm.2005.018234

Renshaw, A., \& Goodwin, P. C. (2016). Injury incidence in a Premier League youth soccer academy using the consensus statement: a prospective cohort study. BMJ Open Sport \& Exercise Medicine, 2(1), e000132. https:/ /doi.org/10.1136/bmjsem-2016-000132
Ríos Azuara, D.; Pérez Flores, D. y Ríos Alcolea, M. (2014). Epidemiología de las lesiones deportivas en países de la unión europea. Revista Internacional de Medicina y Ciencias de La Actividad Física y El Deporte, 14(55), 479-494.

Sánchez Jover, F. y Gómez Conesa, A. (2008). Epidemiología de las lesiones deportivas en baloncesto. Revista Internacional de Medicina y Ciencias de La Actividad Física y El Deporte, 8(32), 270-281. Retrieved from http:// cdeporte.rediris.es/revista/revista $32 /$ artepidemiobc76.htm

Schilaty, N. D., Bates, N. A., Nagelli, C. V., Krych, A. J., \& Hewett, T. E. (2018). Sex-Based Differences of Medial Collateral Ligament and Anterior Cruciate Ligament Strains With Cadaveric Impact Simulations. Orthopaedic Journal of Sports Medicine, 6(4), 2325967118765215. https://doi.org/10.1177/2325967118765215

Tenforde, A. S., Sayres, L. C., McCurdy, M. L., Sainani, K. L., \& Fredericson, M. (2013). Identifying sex-specific risk factors for stress fractures in adolescent runners. Medicine and Science in Sports and Exercise, 45(10), 18431851. https://doi.org/10.1249/MSS.0b013e3182963d75

Toro, V., Guerrero, D., Muñoz, D., Siquier, J., Bartolomé, I., \& Robles, M. C. (2020). Análisis de la incidencia de lesiones y hábitos usados durante el calentamiento en el baloncesto femenino. Retos. Nuevas Tendencias En Educación Física, Deporte y Recreación, 38(38), 159-165.

Villaquirán, A. F., Portilla Dorado, E., \& Vernaza, P. (2016). Caracterización de la lesión deportiva en atletas caucanos con proyección a Juegos Deportivos Nacionales. Universidad y Salud, 18(3), 541-549. https://doi.org/ 10.22267/rus.161803.59

Wahl, C. J., Westermann, R. W., Blaisdell, G. Y., \& Cizik, A. M. (2012). An association of lateral knee sagittal anatomic factors with non-contact ACL injury: Sex or geometry? Journal of Bone and Joint Surgery - Series A, 94(3), 217-226. https://doi.org/10.2106/JBJS.K.00099

Yang, J., Tibbetts, A. S., Covassin, T., Cheng, G., Nayar, S., \& Heiden, E. (2012). Epidemiology of overuse and acute injuries among competitive collegiate athletes. Journal of Athletic Training, 47(2), 198-204. https://doi.org/ 10.4085/1062-6050-47.2.198

Zafra, A. O. (2009). Lesiones y fútbol base: un análisis en dos clubes de la Región de Murcia. RETOS. Nuevas Tendencias En Educación Física, Deporte y Recreación, 2009(16), 63-66.

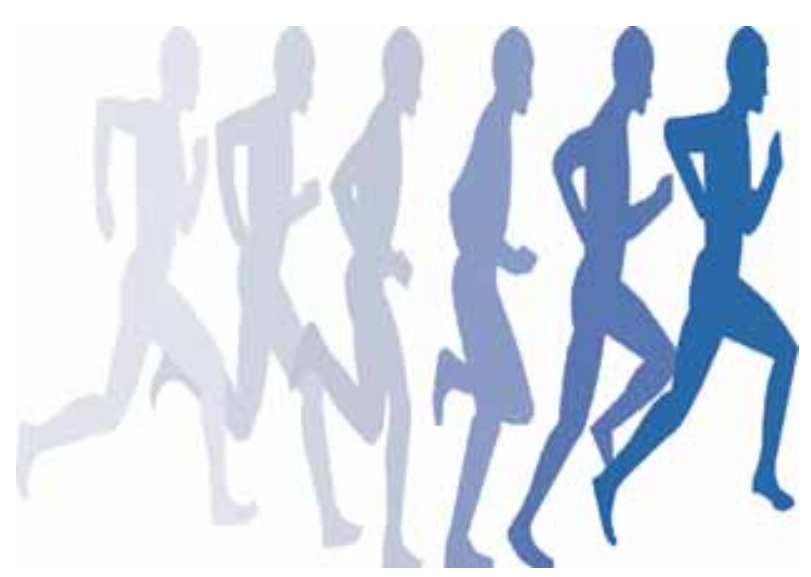

